\title{
PyTMs: a useful PyMOL plugin for modeling common post-translational modifications
}

\author{
Andreas Warnecke ${ }^{1 *}$, Tatyana Sandalova², Adnane Achour ${ }^{2}$ and Robert A Harris ${ }^{1 *}$
}

\begin{abstract}
Background: Post-translational modifications (PTMs) constitute a major aspect of protein biology, particularly signaling events. Conversely, several different pathophysiological PTMs are hallmarks of oxidative imbalance or inflammatory states and are strongly associated with pathogenesis of autoimmune diseases or cancers. Accordingly, it is of interest to assess both the biological and structural effects of modification. For the latter, computer-based modeling offers an attractive option. We thus identified the need for easily applicable modeling options for PTMs.

Results: We developed PyTMs, a plugin implemented with the commonly used visualization software PyMOL. PyTMs enables users to introduce a set of common PTMs into protein/peptide models and can be used to address research questions related to PTMs. Ten types of modification are currently supported, including acetylation, carbamylation, citrullination, cysteine oxidation, malondialdehyde adducts, methionine oxidation, methylation, nitration, proline hydroxylation and phosphorylation. Furthermore, advanced settings integrate the pre-selection of surface-exposed atoms, define stereochemical alternatives and allow for basic structure optimization of the newly modified residues.
\end{abstract}

Conclusion: PyTMs is a useful, user-friendly modelling plugin for PyMOL. Advantages of PyTMs include standardized generation of PTMs, rapid time-to-result and facilitated user control. Although modeling cannot substitute for conventional structure determination it constitutes a convenient tool that allows uncomplicated exploration of potential implications prior to experimental investments and basic explanation of experimental data. PyTMs is freely available as part of the PyMOL script repository project on GitHub and will further evolve.

Keywords: Post-translational modifications, PyMOL plugin, Structural bioinformatics, Modeling, Acetylation, Carbamylation, Citrullination, Oxidations, Malondialdehyde adducts, Nitration

\section{Background}

\section{Post-translational modifications}

Post-translational modifications (PTMs) are chemical alterations that occur after protein synthesis [1,2]. Physiologically, PTMs are selectively introduced by specific, dynamically-regulated enzymes in order to alter the target protein's biochemical properties. PTMs are thus employed to regulate biological processes by altering, for example, protein activity, signaling, localization, conformation, binding or turnover [3-5]. The involvement of PTMs in cellular signaling cascades, particularly kinasemediated phosphorylation, is being extensively researched.

\footnotetext{
* Correspondence: andreas.warnecke@ki.se; robert.harris@ki.se 'Department of Clinical Neuroscience, Karolinska Institutet, Center for Molecular Medicine, Applied Immunology \& Immunotherapy, L8:04, Karolinska Hospital, SE-171 76 Stockholm, Sweden

Full list of author information is available at the end of the article
}

Conversely, pathophysiological PTMs are associated with episodes of oxidative imbalance, and there is a constant cellular endeavor to prevent and repair their occurrence. Unlike physiological PTMs, pathophysiological PTMs typically occur in proximity to the origin of the modifying agent in an uncontrolled fashion. The formation of such pathophysiological PTM on their target proteins may impinge on cellular homeostasis and immune recognition [3-8]. Smoking in particular has been identified as a risk factor with respect to PTMs and inflammation [9-11]. Due to altered immunogenicity and the associated inflammation, pathophysiological PTMs are of particular interest in the context of autoimmune pathogenesis [3-5]. Conversely, the PTMmediated altered immunogenicity of proteins has been suggested as a means of escape from immune surveillance in cancer [7]. 
As implicated above, the effect that a PTM exerts on its carrier protein is highly individual and contextdependent $[3,4,12,13]$. Regarding a protein's activity, modification can be regulatory, resulting in either gainor loss-of-function, or alternatively have no immediate effect - depending largely on the location of the PTM. The latter is argued to have been evolutionarily selected for in the context of Methionine oxidation, in particular in mitochondrial enzymes, because oxidation of the distal 'scavenger' residues protects critical functional sites [14]. Taken together, this highlights the relevance of structural positioning in regard to PTMs.

There are accumulating examples of specific PTMs being recognized by scavenger receptors, complement components and antibodies due to alterations in structure and charge [12,15-17]. One sensible biological function is the accelerated disposal of damaged proteins. Conversely, a classic example is the accumulation of 'foam cells' in atherosclerotic plaques. These are macrophages that have accumulated oxidized low density lipoprotein due to increased uptake mediated by the scavenger receptor CD36 [16]. More recently, complement factor $\mathrm{H}(\mathrm{CFH})$ has been demonstrated to be involved in the disposal of oxidatively modified proteins bearing adducts jointly formed by Malondialdehyde (MDA) and Acetaldehyde (MAA-adduct) [15]. MDA is a highly reactive three-carbon dialdehyde formed during lipid peroxidation. A polymorphism in CFH (HIS instead of TYR at residue 402) is argued to result in a reduced clearance of damaged MAA-modified proteins in the eye, with causative links to age-related macular degeneration [15]. Interestingly, native antibodies from newborns appear to recognize the same modification [17]. The structural nature of this and other such interactions, however, remain to be characterized in detail.

The antigenicity of complement-bound antigen has been demonstrated to increase by several orders of magnitude, as evidenced by co-ligation of the B cell receptor and CD19 bridged by complement component C3dg [18]. This agrees with the reported antigenicity of MDA-adducted proteins $[19,20]$, provided that proinflammatory complement components recognize PTMs and thus 'flag' them for increased immune surveillance. Furthermore, MDA has been experimentally implied in the pathogenesis of animal models of Multiple Sclerosis (MS) [5,21], and there is clinical evidence of elevated lipid peroxidation as a source of MDA in MS patients [22,23].

In fact, most autoimmune diseases have an implied involvement of PTMs, and associated altered peptide ligands (APLs) have been identified [3-5,24,25]. A common mechanistic denominator in autoimmunity (or allergy) is the absence of negative selection of $\mathrm{T}$ or $\mathrm{B}$ cells during education. Such non-tolerized, cross-reactive clones, capable of recognizing 'modified self' or 'cryptic epitopes', are suspected to initiate the break of immunological tolerance $[3,4]$. The key underlying molecular events are the processing of the non-tolerized epitope for presentation, and finally the recognition of this peptide presented within a matching Major Histocompatibility Complex (MHC) by a cross- and/or self-reactive T cell receptor (TCR).

In Rheumatoid Arthritis there is a strong association of specific MHC alleles and their ability to present citrullinated APLs, i.e. epitopes that have undergone enzymatic deimination of arginine residues to citrulline. The molecular basis of this association has only recently been characterized at a structural level [25]. There are accumulating examples of APLs being either superior or inferior epitopes in the context of MHC presentation or TCR recognition $[12,24,25]$. Taken together, these emphasize the requirement for individual structural assessment in the context of immunology and PTMs.

In conclusion, PTMs constitute a complex layer in protein biology and immune recognition. The diversity and context-dependence of PTM-mediated effects calls for individual assessment at a structural level. Furthermore, we identify an unmet need for easily applicable modeling tools in respect to PTMs.

\section{Post-translational modifications in protein models}

As outlined above, it is of increasing interest for researchers to assess the potential impact of PTMs of particular proteins, or to provide structural insight into how an introduced PTM potentially can exert an experimentally observed effect. Recent advances in accounting for PTMs during resolution of $3 \mathrm{D}$ structures have been made [26]. However, resolving a 3D-structure may prove unattractive or infeasible due to financial, technical or time-related reasons. In this case, modeling the PTM in silico provides a rapid alternative. The online resource PhosphoSitePlus (http://www.phosphosite.org/) [27] is able to indicate relevant sites in proteins by mining database entries, but does not include or introduce the PTM unless it is already present in an existing crystal structure. To our knowledge, no simple, freely available tool is available that can easily perform such a task. Manual structure editing is conceivable using the visualization software PyMOL (http://www.pymol.org/), but is likely time-consuming and will thus not provide good flexibility for various models. We therefore developed PyTMs as an applicable PyMOL plugin that enables the straightforward introduction and modeling of a set of PTMs into existing 3D-structures. PyMOL is commonly used, easy to handle and freely available. Advantages of using automated PTM creation include: firstly, standardized and reproducible PTMs; secondly, minimal time-requirement; thirdly, availability of the option to a broader user audience. 
PyTMs currently encompasses ten common PTM categories which are summarized in Figure 1, namely acetylation [8,28,29], carbamylation [9], citrullination [25], cysteine oxidation [30], malondialdehyde adducts $[10,11,16,31-36]$, methionine oxidation [37,38], methylation [39], nitration [6,12-14], proline hydroxylation $[40,41]$ and phosphorylation [42].

\section{Implementation}

\section{PyTMs implementation notes}

We implemented PyTMs as a plugin for the PyMOL Molecular Graphics System (Schrödinger, LLC., http:// www.pymol.org/). The name PyTMs has been chosen as an analogy to PyMOL, Python (https://www.python. org/) and PTMs. PyTMs is written in Python and is open source: the file is freely available as part of the PyMOL script repository, allowing version control and updating (visit http://www.pymolwiki.org/index.php/ Pytms for more information). The first release version is provided as Additional file 1. PyTMs is designed to facilitate the introduction of post-translational modifications into existing 3D-structure models. The intention is to provide a simple research tool that allows making predictions and explaining observations associated with PTMs, yet requiring only basic PyMOL user experience. For this reason we also provided a convenient user interface (cf. Additional file 2).

\section{PyTMs conception}

PyTMs allows modeling of a selected set of common modifications. We used RCSB database (http://www. rcsb.org/) entries containing modified residues as templates where available. Furthermore, the nomenclature of modified residues was adjusted to correspond to this database as far as possible. More detailed information on the implementation of certain PTMs is available in Additional file 3. Though PyTMs employs basic structure editing options already available in PyMOL, the advantage of using PyTMs is the automation of the process, which enables basic users to edit any structure within seconds and currently does not depend on a library.

\section{Basic algorithm}

The basic algorithm of PyTMs follows a pattern that is similar for each individual PTM. First, the input selection is broken down to filter candidate amino acids as determined by the class of PTM and settings. If selected, this will involve a pre-selection of surface-exposed residues, based on a provided solvent-accessible area cutoff (in $\AA^{2}$ ). The subsequent sub-selection of target atoms for modification is automatic, thus the user does not need to provide a specific selection, apart from the object or atom group. Selections or objects can also be chosen from the integrated menu. The candidate residues are filtered for existing modifications and sequentially modified, renamed, adjusted and optionally colored.

For some PTMs, e.g. Malondialdehyde adducts, we implemented a residue-based basic structure optimization. This optimization avoids steric Van-der-Waals (vdW) clashes by rotating the residue into a favorable position. The calculated vdW strain is minimized in the process. For reduction of calculation time the strain calculation is performed locally per residue. There is an option to probe baseline strain increase and thus avoid calculation loops for unfavorable positioning of the original residue. The optimization and/or steric clashes can be visualized in PyMOL (cf. Additional file 4).

\section{Further information}

Practical information, such as a user guide and application notes, are available at the PyTMs wiki page (http:// www.pymolwiki.org/index.php/Pytms) or by accessing each function's specific help, e.g. from the plugin menu. Users have the option of using either the menu and/or the Python function that extend the PyMOL API, e.g. for use in scripts. PyTMs has been developed and tested using the current PyMOL incentive version 1.7, but is compatible with version 1.3. Older versions and opensource PyMOL have minor restrictions, the main one being the lacking support of custom property 'p.PTM' selectors. The overall functionality is, however, otherwise not affected. Modified models can be saved as '.pkl' files, which preserve bonds and valences correctly. We anticipate that additional PTMs and functionality will be added as PyTMs becomes updated in due time.

\section{Application examples}

Here we provide some basic application examples of how PyTMs can be employed to address research questions related to PTMs, which we discuss below in the Results section:

\section{Enzyme inhibition: nitrated HPR1}

The model of hydroxypyruvate reductase 1 (HPR1) from Arabidopsis thaliana was kindly provided by Dr. Francisco J. Corpas [43]. The missing nicotinamide adenine dinucleotide phosphate (NADPH) co-factor was added from the aligned structural homologue Pyrococcus horikoshii hydroxypyruvate reductase [PDB: 2DBQ] and slightly adjusted to fit the cavity without significant steric clashes. Using PyTMs, we introduced nitrations of Tyrosine residues that have previously been experimentally determined to be nitrated [43]. In particular, nitration of TYR198 close to the active site has been demonstrated to inhibit enzyme activity. The steric vdW clashes in HPR and the NADPH co-factor (Figure $2 \mathrm{~B}+\mathrm{C}$ ) were 


\begin{tabular}{|c|c|c|c|c|c|c|c|}
\hline PTM & PyTMs command & p.PTM selector & Target amino acid(s) & N-terminus* & Charge/Polarity effect & Group below & References \\
\hline acetylatation & acetylate & acetylation & Lysine & $\begin{array}{l}\text { Yes, biologically } \\
\text { relevant }\end{array}$ & loss of positive charge & B & {$[8,28,29]$} \\
\hline carbamylation & carbamylate & carbamylation & Lysine & $\begin{array}{c}\text { Yes, } \\
\text { implemented }\end{array}$ & loss of positive charge & D & [9] \\
\hline citrullination & citrullinate & citrullination & Arginine & No & loss of positive charge & $E$ & [25] \\
\hline cysteine oxidation & oxidize_cys & oxidation_cys & $\begin{array}{c}\text { Cysteine } \\
\text { (Selenocysteins) }\end{array}$ & No & increased polarity & G & [30] \\
\hline $\begin{array}{l}\text { malondialdehyde } \\
\text { adducts }\end{array}$ & mda_modify & MDA & Lysine & $\begin{array}{c}\text { Yes, biologically } \\
\text { relevant }\end{array}$ & loss of positive charge & A & {$[10,11,16,31-36]$} \\
\hline $\begin{array}{c}\text { methionine } \\
\text { oxidation }\end{array}$ & oxidize_met & oxidation_met & $\begin{array}{c}\text { Methionines } \\
\text { (Selenomethionines) }\end{array}$ & No & increased polarity & $\mathrm{H}$ & {$[37,38]$} \\
\hline methylation & methylate & methylation & Lysine & $\begin{array}{l}\text { Yes, } \\
\text { implemented }\end{array}$ & increases positive charge & C & [39] \\
\hline nitration & nitrate & nitration & $\begin{array}{l}\text { Tyrosine, } \\
\text { Tryptophan }\end{array}$ & No & increased polarity, lower pKa & 1 & {$[6,12-14]$} \\
\hline $\begin{array}{c}\text { proline } \\
\text { hydroxylation }\end{array}$ & hydroxy_pro & hydroxylation_pro & Proline & No & increased polarity & $\mathrm{F}$ & {$[40,41]$} \\
\hline phosphorylation & phosphorylate & phosphorylation & $\begin{array}{c}\text { Serine, Threonine, } \\
\text { Tyrosine }\end{array}$ & No & introduces negative charge & $J$ & [42] \\
\hline
\end{tabular}

\section{A) MDA adducts on Lysine ( 6 of 48)}

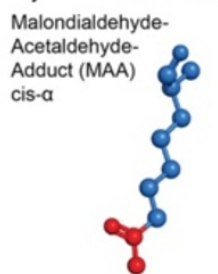

Malondialdehyde-

Acetaldehyde-

Adduct (MAA)

trans- $\beta$

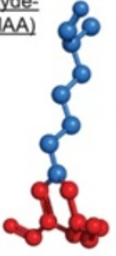

B)Acetylation (Nع, 1 of 1)

\section{Ne-Lysine} Acetylation

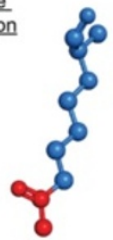

D) Carbamylation ( $\mathrm{N} \varepsilon, 1$ of 1$)$

\section{Ne-Lysine}

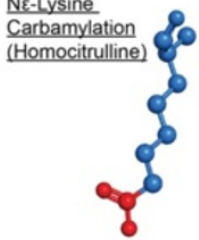

G) Cysteine oxidation (2 of 4)

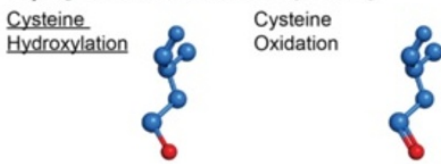

H) Methionine oxidation (2 of 3)

Methionine

Oxidation (R)

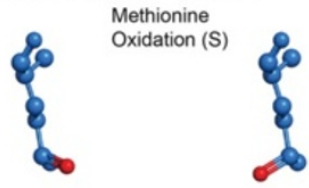

MalondialdehydeAcetaldehyde-

Adduct:

FAAB

fragment

(S)

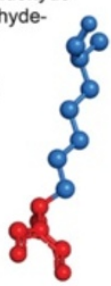

Malondialdehyde-

Adduct:

(trans)

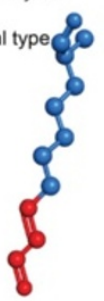

C) Methylation ( $\mathrm{N} \varepsilon, 3$ of 3)

\section{Ne-Lysine}

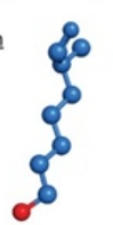

Ne-Lysine

Di-Methylation

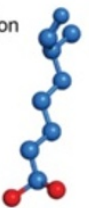

Malondialdehyde-

Adduct:

imine-al type

(trans)

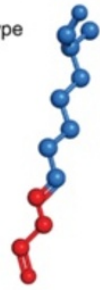

Ne-Lysine

Tri-Methylation

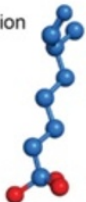

\section{E) Citrullination (1 of 1)}

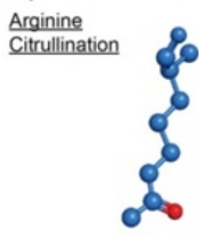

F) Proline hydroxylation (1 of 2)

Hydroxyl-

proline

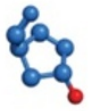

I) Nitration of Tyrosine or Typtophan (1 of 1) | (2 of 5)

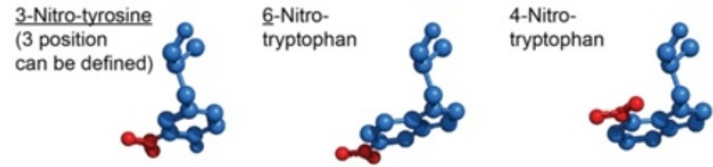

J) Phosphorylation (1 of 1 each)

Phospho-Serine

Phospho-Threonine

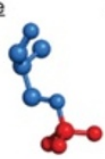

Phospho-Tyrosine

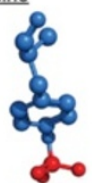

Figure 1 (See legend on next page.) 
(See figure on previous page.)

Figure 1 Overview. This figure gives an overview over all currently covered PTMs that can be introduced in PyMOL using PyTMs. Top: A table of the possible modifications in alphabetical order. PTM = post-translational modification. The columns from left to right contain: name of modification, the PyTMs command that is used to introduce it in the PyMOL API (when not using the GUI menu), a custom property selector name through which the modification can be selected in PyMOL (requires incentive PyMOL version 1.7 or higher), the target amino acid, information on whether $\mathrm{N}$-termini can be modified using the respective function $\left({ }^{*}=\right.$ modifiable, excluding Proline), a summary of charge effects, a group indicator (see below) and lastly selected references. Bottom: A depiction of representative modifications from all classes, grouped by amino acid and modification type. Note that the individual panels A-J correspond to the 'group below' column of the above table. All residues are aligned by the amino acid head, the terminal carboxyl group facing towards the viewer. The base is colored blue and the PTMs in red. Hydrogens are omitted. Groups are labeled as in the table above. For each category the displayed and total number of variants are indicated. The respective defaults for categories are underlined. Configurational diastereomers are indicated with the R or S nomenclature.

visualized using an adapted version of the PyMOL script 'show_bumps' (available on the PyMOL wiki), which has been integrated within PyTMs. This visualization of vdW clashes can be performed directly in conjunction with modification. Alternatively, clashes can be visualized in unmodified protein or retrospectively by using a dedicated function.

\section{SWISS modeling and multiple modifications: oxidation of hERG1}

As the structure of human ether à go-go-related gene 1 (hERG1, $\mathrm{K}_{\mathrm{V}} 11.1, \mathrm{KCNH} 2$ ) is only partially available, we applied SWISS homology modeling [44] using HCN2-I [PDB: 3BPZ] [45] as template to generate a joined model of the hERG1 intracellular C-terminal region (residues 667-865), including the C-linker and regulatory domains. Using PyTMs we oxidized Cysteines and Methionines, either in R- or S- configuration, and superimposed the results for comparison (Figure 3).

\section{MAA-modification of Bovine Serum Albumin surface- exposed lysines}

Here we exemplify the integration of PyTMs with subselections and the possibility to predict surface-restricted modifications, and basic optimization. Pre-selection of surface-exposed atoms is managed by a cutoff using the calculated solvent-accessible surface area for each atom. This pre-selection is set by a user-provided cutoff and is

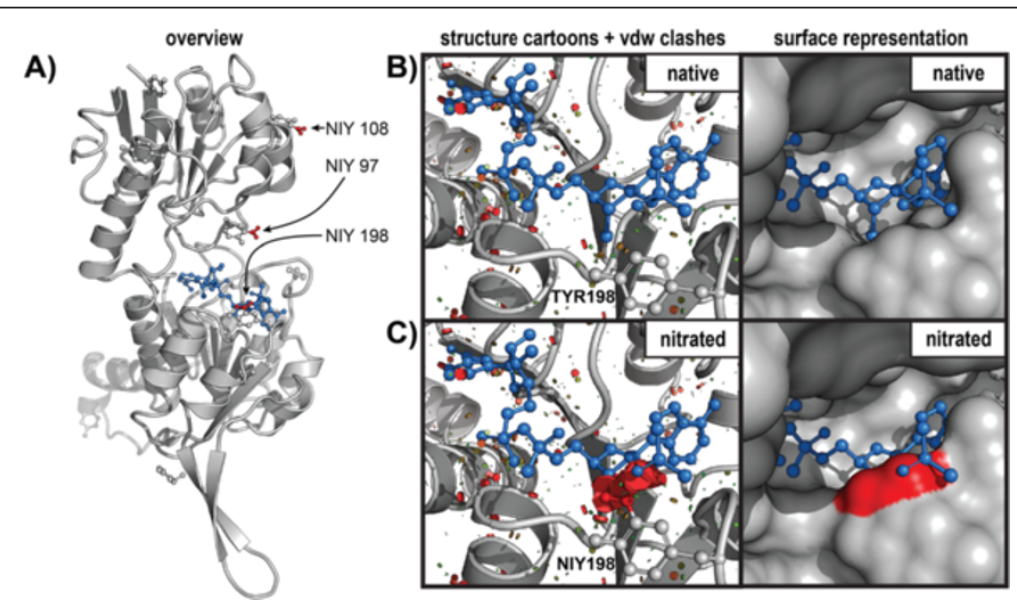

Figure 2 Example - inhibition of HPR1 by nitration. This figure is an application example of using PyTMs in the setting of enzyme inhibition. Briefly, the model of hydroxypyruvate reductase 1 from Arabidopsis thaliana was nitrated at three specific Tyrosine residues using PyTMS as described in the implementation section. This enzyme has previously been demonstrated to be nitrated at these residues and thereby functionally inhibited. $\mathrm{NIY}=$ Nitrotyrosine. $\mathbf{A})$ An overview of the nitrated enzyme monomer is depicted in secondary structure cartoon representation. Additionally, the locations of (Nitro-)Tyrosine residues have been highlighted in stick representation. The nitro groups in residues 97,108 and 198 are colored red. The co-factor (NADPH) in stick representation is colored blue. B + C) A close-up view of the co-factor (NADPH) binding site in the enzyme. B: native; C: nitrated. Left: Representation as cartoon with the (nitrated) TYR198. Steric clashes based on vdW overlap are represented by the colored discs. The increasing size and redness of the discs correlate with stronger vdW strain. The native enzyme does not have significant steric clashes. The nitration, however, introduces significant steric clashing with the co-factor and occludes the binding site. Right: identical view as in the left image, but depicted as surface with the extent of the nitration colored red. In conclusion, the denied binding of co-factor, mainly due to nitration at TYR198, is the structural basis for impaired enzyme activity. 


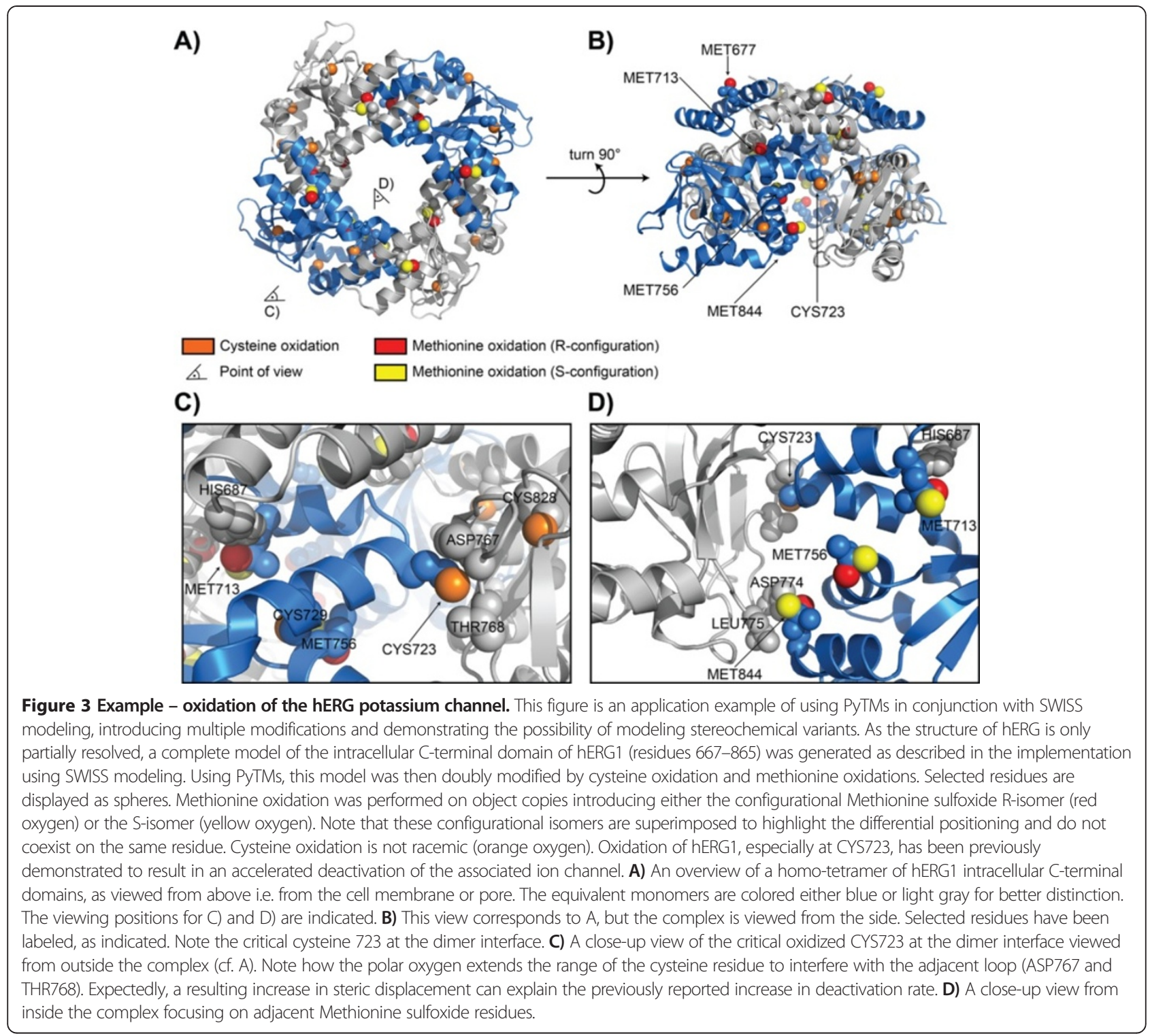

integrated in PyTMs. The original procedure is described on the PyMOL wiki ('findSurfaceResidues'). Here, we restricted modification to surface-exposed Lysine epsilon amine groups in a model of Bovine Serum Albumin (BSA, [PDB: 4F5S]), using a cutoff of $25 \AA^{2}$. This cutoff yielded a reasonable selection of exposed atoms that were modified using PyTMs. We introduced the default Malondialdehyde-acetaldehyde (MAA) adduct (cf. Additional file 3). An optimization level of 3 was applied to account for potential steric overlap of the bulky adducts.

\section{Altered peptide ligands - MHC complexes}

Crystal structures of the immunodominant lymphocytic choriomeningitis virus (LCMV)-derived epitope gp34 in complex with a murine Major Histocompatibility Complex (MHC, $\mathrm{H}-2 \mathrm{~K}^{\mathrm{b}}$ ) have previously been determined for the native epitope [PDB: 3ROO] and a nitrated escape variant [PDB: 3ROL] [24]. Starting with the native unmodified variant we nitrated the peptide ligand in silico and sterically refined the structure. First we tested alternative ortho-positions for the nitration (rotamer of 3-Nitrotyrosine). We thereafter chose alternative backbone-dependent rotamers for GLU152 in the MHC side chain and TYR116 in the beta-sheet of the MHC heavy chain peptide-binding cleft. These two adaptations were expected to accommodate the nitro group without significant steric clashes. Finally, we aligned the resulting models with the experimentally resolved structure containing the nitrated epitope. The 
alignment demonstrated that the model created using PyTMs corresponded well to the previously determined crystal structure [24].

\section{Results and discussion \\ Straightforward modeling of post-translational modifications}

The purpose of PyTMs is to facilitate modeling of PTMs. There is a steady interest from various fields in the impact of PTMs and hence a need for tools that enable their prediction and analysis. Although basic structure editing options are available in the PyMOL base package, we identified two hurdles. Firstly, manual editing requires additional user experience and secondly, it can be laborious even when using libraries. We therefore set out to facilitate the procedure by developing a plugin.

We implemented ten different PTMs as listed in Figure 1. These cover a large ensemble of common PTMs, all of which are well-studied and implicated either in important physiological processes or in disease settings (cf. Figure 1). Here we provide a selection of examples and discuss the overall applicability of PyTMs as a plugin tool.

\section{Application example: enzyme inhibition in nitrated HPR1} We searched the literature for examples of PTMs affecting enzymatic activity. For instance, Corpasa et al. demonstrated that nitration of TYR198 in hydroxypyruvate reductase 1 from Arabidopsis thaliana results in enzyme inhibition [43]. Using site-directed mutagenesis the authors confirmed that nitration of TYR198 in the active site is responsible for the loss of function, but they did not include nitrations in the structural model. Using the PyTMs plugin we introduced nitration at the relevant sites and investigated the model in further detail. Consistent with the previously reported observations, we demonstrate that nitration of TYR198 obstructs the cofactor binding site, providing a structural explanation for reduced enzyme activity (Figure 2 ).

This example demonstrates that PyTMs can be utilized to help explain experimental observations on the basis of a structural model and suggest that it can also be used to predict such loss of function.

\section{Application example: multiple oxidation of hERG1}

The human ether à go-go-related gene 1 hERG1 (K 11.1 , $\mathrm{KCNH} 2$ ) encodes a potassium channel infamous for offtarget drug side-effects that result in cardiac arrhythmias. Structurally hERG1 resembles other members of hyperpolarization-activated, cyclic nucleotide-modulated (HCN) channels [45]. The pore-forming transmembrane domains are connected to an intracellular C-linker region and a regulatory ligand-binding domain. Both the
C-linker and the ligand-binding domain contain residues that are involved in homo-tetramerization of the intracellular domains. This structured tetramerization is regarded as the underlying mechanism towards opening the associated potassium channel upon ligation [45]. The intracellular region, in particular the C-linker, is sensitive to oxidation of Methionine [46] and/or Cysteine [30] residues. These oxidations account for an accelerated channel deactivation $[30,46]$.

Our extended model in Figure 3 supports the previously reported conclusions. The generated model supports a critical role for oxidation of CYS723 at the dimer interface, which is consistent with a faster deactivation of hERG1 following oxidation due to increased structural strain. The same argument holds for Methionine 844. The oxidized MET844 has proximity to the dimer interface and sterically clashes with ASP774 and LEU775 of the neighboring chain. As MET713 or MET756 do not have immediate proximity to neighboring subunits, the inhibitory effect may involve rearrangement of the associated helices upon oxidation of these residues, and therefore a more indirect mode of inhibition. Note that the alternative configurational isomers (R:red, S: yellow) may have unequal impact in this respect.

Based on this model the critical role of CYS723 in the susceptibility of hERG1 to oxidation derives from its open accessibility and positioning at the dimer interface. The equivalent explanation is valid for MET844. For the remaining Methionine sulfoxides the inhibition is expected to be less direct and may involve rearrangement of the associated C-linker helices. Hence, several $\mathrm{Me}$ thionine residues may require oxidation to confer the same degree of inhibition as oxidation of CYS723 or MET 844 alone.

This example alludes to several modeling aspects. First, it exemplifies the use of homology modeling [44] in case a resolved structure is unavailable. Secondly, the model is an example of introducing several different PTMs into one protein model. In fact, the only condition for consecutive modification in silico using PyTMs is that the target atom is still available. For instance, it is possible to co-modify an N-terminal Tyrosine by phosphorylation, nitration and $\mathrm{N}$-terminal acetylation (see also graphical abstract, Additional file 5). This may not necessarily be biologically relevant, as nitration has been demonstrated to impede subsequent enzymatic phosphorylation [47], but this is allowed within the program. Finally, this example addresses modeling of diastereomers [48]. We implemented biologically relevant diastereomers for Methionine sulfoxide, Cysteine-s-dioxides and the 2-formyl-3-(alkylamino)-butanal (FAAB fragment) adduct of Malondialdehyde. Hydroxyl-proline is technically chiral but biologically always present in a 4-R configuration, since this is an enzymatic modification. 
However, the option for a 4-S variant exists in PyTMs. As diastereomers of Methionine sulfoxide are repaired by distinct enzymes [38] we suspect that additional biomolecules such as antibodies, T-cell receptors, complement components and scavenger receptors also discriminate stereospecific variants [48].

Notably, this example also indicates a current limitation of the plugin, namely a disregard for potential global conformational changes induced by PTMs. Although some manual local refinement can be informative, larger rearrangements will become increasingly artificial and unreliable. Conformational changes that affect large or distal areas will be especially problematic. With this in mind conventional structure determination, although laborious, will yield more conclusive results. Here we point out SWISS modeling [44] as a previously available resource that can be applicable in case of unavailable crystal structures.

In summary, it is possible to induce several modifications in one model and specify stereospecific variants. The resulting model should, however, not be overinterpreted as potential global conformational changes are not handled by the software. Nonetheless, it is possible to explain experimental data or make predictions at a detailed structural level using PyTMs when respecting these caveats.

\section{Application example: MDA-modification of Bovine Serum Albumin surface-exposed Lysines}

A critical factor that influences the likelihood of modification is accessibility of the target atom. Non-selective oxidative modifications, such as Malondialdehyde (MDA) derived from lipid peroxidation, will primarily target the most accessible residues while buried residues are likely spared. Using PyTMs we selected only surface-exposed epsilon-amines (area cutoff: $25 \AA^{2}$ ) in a model of BSA and introduced Malondialdehyde-acetaldehyde (MAA) adducts. We implemented a basic vdW strain optimization for this PTM category as many adducts are comparatively bulky and tend to sterically clash with adjacent residues. Applying optimization rotates the modified Lysine to a more favorable rotamer, but requires some calculation time. We implemented that this optimization can be animated in PyMOL (cf. Additional file 4). Additional information on the implementation of MDA adducts is provided in the Additional file 2 [48,49].

Figure 4 displays MAA-modified BSA in which the surface-accessible amines have been modified (75 of 118 Lysines). The pre-selection noticeably restricted modification to surface residues while inaccessible Lysines remained unmodified (Figure $4 \mathrm{~B}$ ). Indeed, buried residues are most likely protected from modification within the protein, unless it unfolds in the process. Unlike the first example, in which the nitrations were detected by
Mass-Spectrometry prior to modeling, this example is predictive. Notably, should Mass-Spectrometry detect modified residues at unexpected locations, this may indicate that the protein becomes unfolded during the modification process. In relation to topology we were unable to assess the associated charge impact of modification. The limitation is currently that force field applications such as the Adaptive Poisson-Boltzmann Solver (APBS) [50] currently only support standard amino acids. This aspect may be addressed in the future, but will require the generation of custom force fields for PTM amino acids. Experimental data indicates that increasing MAA modification gradually eliminates positive charge and thus alters the migration of proteins in native or isoelectric focusing gels ([36] and data not included).

In summary, we conclude that restricting modification to experimentally defined or accessible atoms are feasible approaches to improve model accuracy. Optimally, each model should be generated according to supporting experimental data at hand or be experimentally validated accordingly. We point out that PyTMs could for instance be used to introduce missing PTMs for structure information retrieved from PhosphoSitePlus [27].

\section{Application example: MHC-restricted peptide epitopes}

Modifications can be introduced into single amino acids, peptides or whole proteins using PyTMs. In the context of altered peptide ligands (APLs) and Major Histocompatibility Complexes (MHCs), modeling can be useful to explain or predict whether an APL can be presentable by a respective $\mathrm{MHC}$ allele and/or how the introduced PTM and APL may be oriented.

Herein we made use of a pair of crystal structures of murine $\mathrm{H}-2 \mathrm{~K}^{\mathrm{b}} \mathrm{MHCs}$ in complex with the lymphocytic choriomeningitis virus (LCMV)-derived immunodominant epitope gp34, without or with nitration [24]. The nitrated APL is an escape variant and exhibits significantly reduced binding affinity [24]. Using the native complex we nitrated the Tyrosine at position 3 in gp34 and performed minor local refinements to accommodate the APL.

As described in Figure 5D, we concluded that the orientation of the ortho-position, i.e. the rotamer of Nitro-tyrosine, is a factor that affects the affinity to the MHC. Steric clashes dictate that the nitro group only fits into the groove when facing away from the MHC helix into the groove. This problem does not occur for the Tyrosine itself. Furthermore, this positioning requires structural adaptation of GLU152. Consequently, the hydrogen bonding is altered for the nitrated gp34. The steric displacement of GLU152 breaks a hydrogen bond between this residue and the Nitrotyrosine hydroxyl group (Figure $5 \mathrm{~B}$ ). This loss is partially compensated for by TYR116 and GLN114 forming hydrogen bonds with the nitro group inside the 


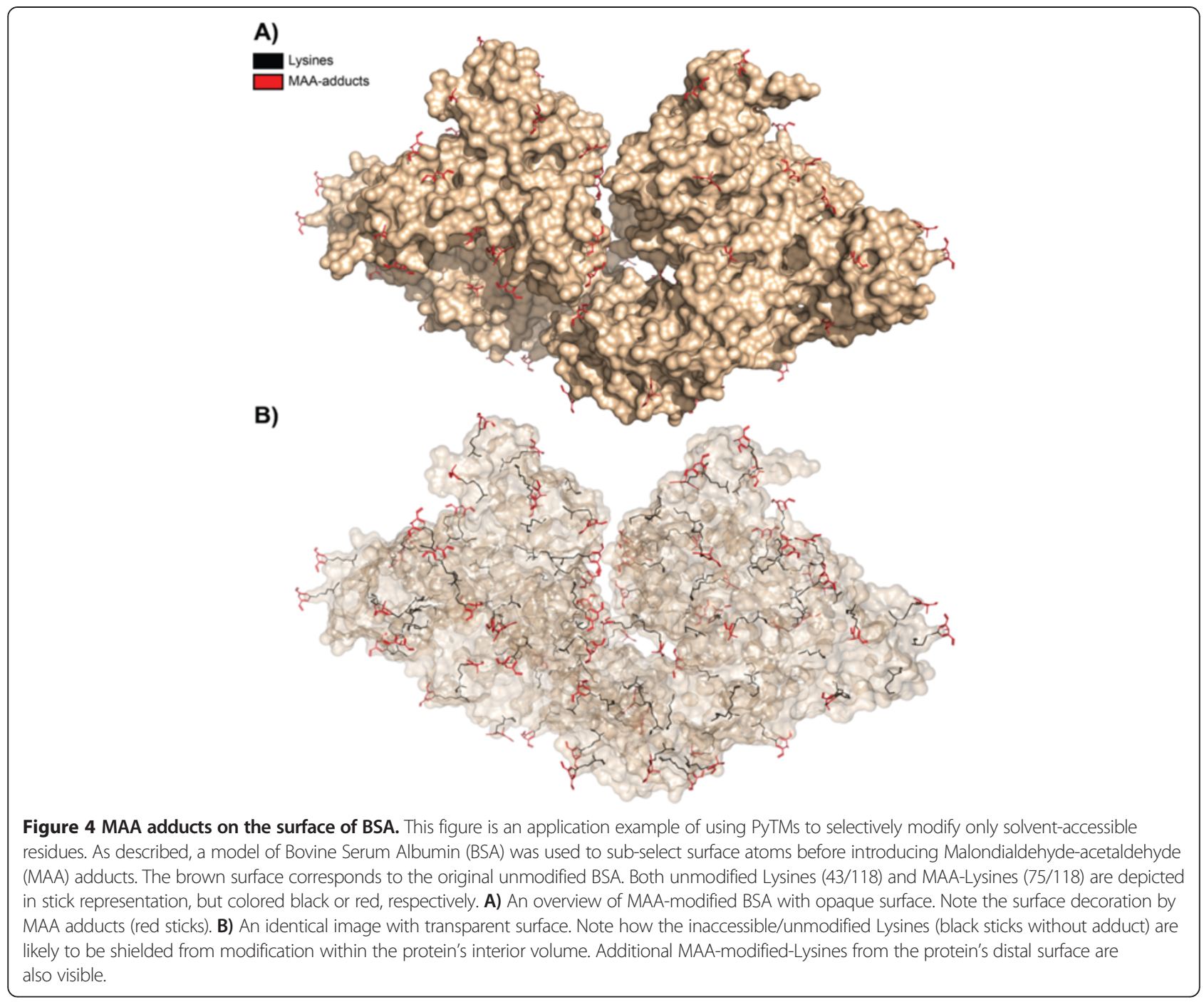

groove. Taken together, this provides an explanation for the reduced binding capacity observed for the nitrated peptide. A final comparison of the refined model to the existing crystal structure with nitrated APL exhibited a striking similarity (Figure 5B vs. 5C).

Furthermore, in Additional file 3 we made use of the same model but introduced alternative PTMs, namely Nterminal acetylation (p1ACE-ALA) and/or methionine oxidation (p8SME). Based on the putative steric clashing with either the MHC rim (p1ACE-ALA) or the anchoring pocket (p8SME), we predict that these modifications will negate binding of the APL to $\mathrm{H}-2 \mathrm{~K}^{\mathrm{b}}$. The acetylated peptide is too long to fit the confinements of the MHC class I groove, and the anchoring pocket cannot properly accommodate the oxidized methionine. In summary, modeling of MHC-associated APLs is possible and yields predictive results within a reasonable frame of confidence. Larger global conformational changes, however, cannot be accounted for using this approach.
We conclude that modeling of APLs can be a useful approach prior to experimental investments. Furthermore, it can also be applicable to predict whether a PTM is likely to be in contact with the MHC and/or a corresponding $\mathrm{T}$-cell receptor.

\section{Conclusions}

\section{Perspective and future development}

To summarize, we have presented several examples of how modeling using PyMOL and PyTMs can be applied to straightforwardly address various research questions related to PTMs. In either predictive or explanatory settings, modeling constitutes a rapid approach to investigate structural implications. The advantages of using a plugin to introduce PTMs include standardized generation of PTMs, rapid time-to-result and facilitated user control. In addition, modeling is an option to maximize cost-efficiency. 


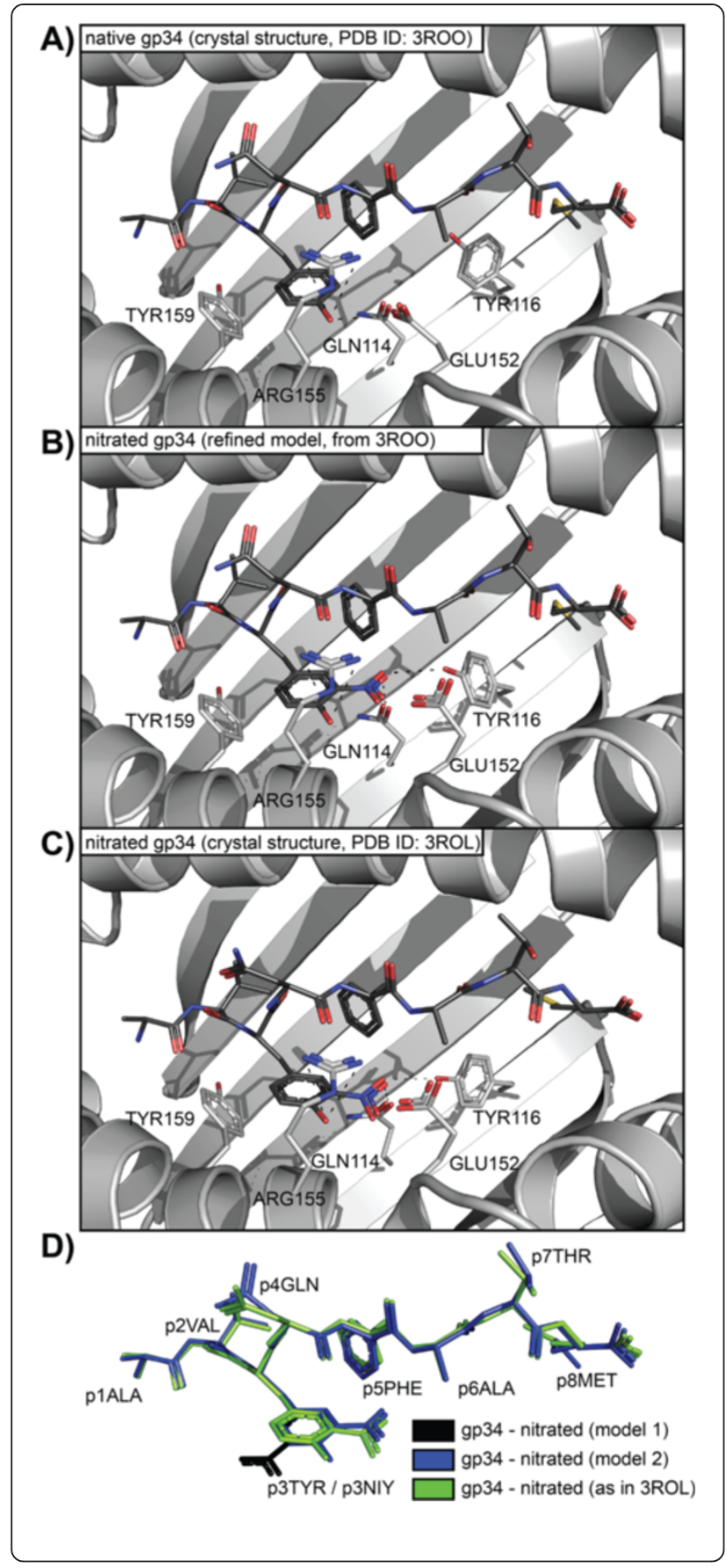

Figure $\mathbf{5}$ MHC-related applications. In this figure we tested the applicability of PyTMs with respect to altered peptide ligands (APLs). As described in the implementation, we started with the crystal structure of a native gp34 LCMV epitope complexed within the mouse MHC class I molecule $\mathrm{H}-2 \mathrm{~K}^{\mathrm{b}}$. We introduced nitration into the p3TYR residue and refined the structure locally to resolve resulting clashes. The results were aligned and compared to the experimentally determined crystal structure. A) Orientation of the native gp34 in the resolved crystal structure [PDB: 3ROO]. The view is focused on the peptide-binding cleft. B) The refined model of a nitrated gp34 APL derived from the same crystal structure. Alternative backbone-dependent rotamers for GLU152 (steric displacement) and TYR116 (hydrogen bonding) have been chosen to accommodate this APL. C) The aligned gp34 APL and MHC pocket from the experimentally resolved crystal structure [PDB: 3ROL]. The essential adaptations are surprisingly similar. We therefore conclude that modeling APLs can be a valid predictive approach. However, the ligation of this APL induces more pronounced and global conformational changes that cannot be accounted for by local refinement. D) Relative positioning of the aligned peptides. Black and blue: Two nitrated gp34 epitopes according to modeling which correspond to $180^{\circ}$ rotamers of each other. The first variant (model 1, black) clashed significantly with the helix of the MHC and TYR159, a feature that may contribute to the reduced affinity of the nitrated epitope (complete model not shown). The alternatively positioned model 2 was used for the modeling above and fits significantly better, especially after local refinement. Note how the orientation of this APL is essentially identical to that of the experimentally resolved variant (green).

We identified two current limitations of the plugin, namely the incompatibility of non-standard residues with tested charge force field applications and a limited ability to assess changes in global conformation. Many PTMs are associated with drastic alterations in the charge pattern, rather than steric blocking, citrullination being a prime example. In concordance with this, conformational changes are probably a major mechanism through which certain PTMs mediate their effects. As both aspects are limited in PyTMS, this may restrict the interpretability of resulting models, unless well supported by experimental observations. Resolving these shortcomings presents a future challenge. Bearing this in mind, we support the reasonable application of in silico modeling alongside conventional structure determination.

In conclusion, PyTMs is a free, user-friendly and very convenient plugin for PyMOL and can be applied to straightforwardly address research questions connected to PTMs. We hope to address the discussed challenges over time and further extend the applicability with additional PTMs.

\section{Availability and requirements}

Project name(s): PyTMs, PyMOL script repository.

Project homepage:

http://www.pymolwiki.org/index.php/Pytms.

https://github.com/Pymol-Scripts/Pymol-script-repo. 
Operating systems: Platform independent.

Programming language: Python (https://www.python.org/). Other requirements: PyMOL (http://www.pymol.org/). License: GNU General Public License, version 2 (GPL-2.0).

\section{Additional files}

Additional file 1: PyTMs. The first release version of PyTMs (for deposit purposes); This file should optimally renamed to pytms.py and placed inside the 'plugins' folder of the 'PyMOL-script-repo' when installed. Note that PyTMs will be updated/maintained on the hompage site: http:// www.pymolwiki.org/index.php/Pytms. Here you should find the latest version.

Additional file 2: PyTMs supplementary images. Supporting images, PyTMs menu screenshot and PTM overviews.

Additional file 3: PyTMs supplementary online information. Supporting information on the implementation of MDA/MAA adducts, supporting figures.

Additional file 4: This is an example of the residue-based optimization in PyTMs. The optimization resolves sterical clashes (represented by red discs) by positioning the adducted residue in a more favorable position, based on minimal local strain. This examples shows a test protein (PDB:1hpv) with malondialdehyde-acetaldehyde (MAA) adducts (red). The animated gif file can be viewed in a web-browser.

Additional file 5: PyTMs - graphical abstract.png. Graphical abstract. In this example we modified a generic peptide (MKCRYKWKSTP) to display a selection of different modifications, as indicated. Colors: original carbons $=$ white, hydrogens = light grey, nitrogens = blue, oxygens = red, sulfur= yellow, phosphorus = orange, adducted carbons = dark grey; MDA/ MAA = malondialdehyde/ malondialdehyde-acetaldehyde.

\section{Abbreviations}

API: Application programming interface; APL: Altered Peptide Ligand; MAA: Malondialdehyde Acetaldehyde; MDA: Malondialdehyde; MHC: Major Histocompatibility Complex; PTMs: Post-translational modifications; PyTMs: Name of the PyMOL plugin; an analogy to PyMOL and PTMs; TCR: T cell receptor.

\section{Competing interests}

The authors declare that they have no competing interest.

\section{Authors' contributions}

AW developed PyTMs, modeled the examples, and wrote the article. TS tested PyTMs and gave inputs for improvement. AA \& RAH participated in coordination, design and drafting the article. All authors read and approved the final manuscript.

\section{Authors' information}

AW and RAH are applied immunologists with particular interest in autoimmunity. TS and AA are structural biologists with particular competence in MHC complexes. This article is an interdisciplinary effort bridging structural bioinformatics to applications especially within immunology. We regard the impact of PTMs on immunological processes as being highly context-dependent and there is a need for assessment on an individual, structural level. PyTMs is a tool that can be employed to explore such questions, but we envision its applicability for other purposes, e.g. in the field of signaling.

\section{Acknowledgements}

AW expresses his appreciation to Thomas Holder from Schrödinger for feedback on the PyTMs script, and to contributors to the PyMOL wiki. This article was funded by the Swedish Medical Research Council, BarnDiabetesFonden, DiabetesFonden and Karolinska Institutet. AW was supported by a StratNeuro (2013) and NHR fonden (Neuro Sweden, 2014). The funding bodies had no influence on design, execution or analysis of this work.

\section{Author details}

'Department of Clinical Neuroscience, Karolinska Institutet, Center for Molecular Medicine, Applied Immunology \& Immunotherapy, L8:04, Karolinska Hospital, SE-171 76 Stockholm, Sweden. ${ }^{2}$ Department of Medicine Solna, Science for Life Laboratory, Karolinska Institutet, Stockholm, Sweden.

Received: 25 July 2014 Accepted: 30 October 2014

Published online: 28 November 2014

\section{References}

1. Bischoff R, Schluter H: Amino acids: chemistry, functionality and selected non-enzymatic post-translational modifications. J Proteomics 2012, 75(8):2275-2296.

2. Butterfield DA, Gu L, Di Domenico F, Robinson RA: Mass spectrometry and redox proteomics: applications in disease. Mass Spectrom Rev 2014, 33(4):277-301.

3. Anderton SM: Post-translational modifications of self antigens: implications for autoimmunity. Curr Opin Immunol 2004, 16(6):753-758.

4. Doyle HA, Mamula MJ: Posttranslational modifications of self-antigens. Ann N Y Acad Sci 2005, 1050(1):1-9.

5. Harris RA, Amor S: Sweet and sour - oxidative and carbonyl stress in neurological disorders. Cns Neurol Disord Dr 2011, 10(1):82-107.

6. Jones LH: Chemistry and biology of biomolecule nitration. Chem Biol 2012, 19(9):1086-1092.

7. Nagaraj S, Gupta K, Pisarev V, Kinarsky L, Sherman S, Kang L, Herber DL, Schneck J, Gabrilovich DI: Altered recognition of antigen is a mechanism of CD8+ T cell tolerance in cancer. Nat Med 2007, 13(7):828-835.

8. He XL, Radu C, Sidney J, Sette A, Ward ES, Garcia KC: Structural snapshot of aberrant antigen presentation linked to autoimmunity: the immunodominant epitope of MBP complexed with I-Au. Immunity 2002, 17(1):83-94

9. Wang Z, Nicholls SJ, Rodriguez ER, Kummu O, Horkko S, Barnard J, Reynolds WF, Topol EJ, DiDonato JA, Hazen SL: Protein carbamylation links inflammation, smoking, uremia and atherogenesis. Nat Med 2007, 13(10):1176-1184.

10. Freeman TL, Haver A, Duryee MJ, Tuma DJ, Klassen LW, Hamel FG, White RL, Rennard SI, Thiele GM: Aldehydes in cigarette smoke react with the lipid peroxidation product malonaldehyde to form fluorescent protein adducts on lysines. Chem Res Toxicol 2005, 18(5):817-824

11. McCaskill ML, Kharbanda KK, Tuma DJ, Reynolds JD, DeVasure JM, Sisson JH, Wyatt TA: Hybrid malondialdehyde and acetaldehyde protein adducts form in the lungs of mice exposed to alcohol and cigarette smoke. Alcohol Clin Exp Res 2011, 35(6):1106-1113.

12. Souza JM, Peluffo G, Radi R: Protein tyrosine nitration-functional alteration or just a biomarker? Free Radic Biol Med 2008, 45(4):357-366.

13. Ohmori $\mathrm{H}$, Kanayama N: Immunogenicity of an inflammation-associated product, tyrosine nitrated self-proteins. Autoimmun Rev 2005, 4(4):224-229.

14. Castro L, Demicheli V, Tortora V, Radi R: Mitochondrial protein tyrosine nitration. Free Radic Res 2011, 45(1):37-52.

15. Weismann D, Hartvigsen K, Lauer N, Bennett KL, Scholl HP, Charbel Issa P, Cano M, Brandstatter H, Tsimikas S, Skerka C, Superti-Furga G, Handa JT, Zipfel PF, Witztum JL, Binder CJ: Complement factor $\mathrm{H}$ binds malondialdehyde epitopes and protects from oxidative stress. Nature 2011, 478(7367):76-81

16. Weismann D, Binder CJ: The innate immune response to products of phospholipid peroxidation. Biochim Biophys Acta 2012, 1818(10):2465-2475

17. Wang C, Turunen SP, Kummu O, Veneskoski M, Lehtimaki J, Nissinen AE, Horkko S: Natural antibodies of newborns recognize oxidative stressrelated malondialdehyde acetaldehyde adducts on apoptotic cells and atherosclerotic plaques. Int Immunol 2013, 25(10):575-587.

18. Cherukuri A, Cheng PC, Pierce SK: The role of the CD19/CD21 complex in $B$ cell processing and presentation of complement-tagged antigens. J Immunol 2001, 167(1):163-172.

19. Willis MS, Klassen LW, Tuma DJ, Sorrell MF, Thiele GM: Adduction of soluble proteins with malondialdehyde-acetaldehyde (MAA) induces antibody production and enhances T-cell proliferation. Alcohol Clin Exp Res 2002, 26(1):94-106.

20. Willis MS, Thiele GM, Tuma DJ, Klassen LW: T cell proliferative responses to malondialdehyde-acetaldehyde haptenated protein are scavenger receptor mediated. Int Immunopharmacol 2003, 3(10-11):1381-1399. 
21. Wallberg M, Bergquist J, Achour A, Breij E, Harris RA: Malondialdehyde modification of myelin oligodendrocyte glycoprotein leads to increased immunogenicity and encephalitogenicity. Eur J Immunol 2007, 37(7):1986-1995.

22. Gonzalo H, Brieva L, Tatzber $F$, Jové $M$, Cacabelos D, Cassanyé A, LanauAngulo L, Boada J, Serrano JC, González C, Hernández L, Peralta S, Pamplona $\mathrm{R}$, Portero-Otin M: Lipidome analysis in multiple sclerosis reveals protein lipoxidative damage as a potential pathogenic mechanism. J Neurochem 2012, 123(4):622-634.

23. Ferretti $\mathrm{G}$, Bacchetti T: Peroxidation of lipoproteins in multiple sclerosis. J Neurol Sci 2011, 311(1-2):92-97.

24. Madhurantakam C, Duru AD, Sandalova T, Webb JR, Achour A: Inflammation-associated nitrotyrosination affects TCR recognition through reduced stability and alteration of the molecular surface of the MHC complex. PLoS One 2012, 7(3):e32805.

25. Scally SW, Petersen J, Law SC, Dudek NL, Nel HJ, Loh KL, Wijeyewickrema LC, Eckle SB, van Heemst J, Pike RN, McCluskey J, Toes RE, La Gruta NL, Purcell AW, Reid HH, Thomas R, Rossjohn J: A molecular basis for the association of the HLA-DRB1 locus, citrullination, and rheumatoid arthritis. J Exp Med 2013, 210(12):2569-2582.

26. Nagata $K$, Randall A, Baldi P: Incorporating post-translational modifications and unnatural amino acids into high-throughput modeling of protein structures. Bioinformatics 2014, 30(12):1681-1689.

27. Hornbeck PV, Kornhauser JM, Tkachev S, Zhang B, Skrzypek E, Murray B, Latham V, Sullivan M: PhosphoSitePlus: a comprehensive resource for investigating the structure and function of experimentally determined post-translational modifications in man and mouse. Nucleic Acids Res 2012, 40(Database issue):D261-D270.

28. Zhao $S, X u$ W, Jiang $W$, Yu W, Lin Y, Zhang T, Yao J, Zhou L, Zeng Y, Li H, Li Y, Shi J, An W, Hancock SM, He F, Qin L, Chin J, Yang P, Chen X, Lei Q, Xiong Y, Guan KL: Regulation of cellular metabolism by protein lysine acetylation. Science 2010, 327(5968):1000-1004.

29. Choudhary C, Kumar C, Gnad F, Nielsen ML, Rehman M, Walther TC, Olsen $\mathrm{JV}$, Mann M: Lysine acetylation targets protein complexes and coregulates major cellular functions. Science 2009, 325(5942):834-840.

30. Kolbe K, Schonherr R, Gessner G, Sahoo N, Hoshi T, Heinemann SH: Cysteine 723 in the C-linker segment confers oxidative inhibition of hERG1 potassium channels. J Physiol 2010, 588(Pt 16):2999-3009.

31. Tuma DJ, Thiele GM, Xu D, Klassen LW, Sorrell MF: Acetaldehyde and malondialdehyde react together to generate distinct protein adducts in the liver during long-term ethanol administration. Hepatology 1996, 23(4):872-880

32. Tuma DJ, Kearley ML, Thiele GM, Worrall S, Haver A, Klassen LW, Sorrell MF: Elucidation of reaction scheme describing malondialdehyde - acetaldehyde - protein adduct formation. Chem Res Toxicol 2001, 14(7):822-832.

33. Slatter DA, Murray M, Bailey AJ: Formation of a dihydropyridine derivative as a potential cross-link derived from malondialdehyde in physiological systems. FEBS Lett 1998, 421(3):180-184.

34. Itakura K, Uchida K, Osawa T: A novel fluorescent malondialdehyde-lysine adduct. Chem Phys Lipids 1996, 84(1):75-79.

35. Uchida K, Sakai K, Itakura K, Osawa T, Toyokuni S: Protein modification by lipid peroxidation products: formation of malondialdehyde-derived $\mathrm{N}$ (epsilon)-(2-propenol)lysine in proteins. Arch Biochem Biophys 1997, 346(1):45-52.

36. Uchida K: Lipofuscin-like fluorophores originated from malondialdehyde. Free Radic Res 2006, 40(12):1335-1338

37. Levine RL, Moskovitz J, Stadtman ER: Oxidation of methionine in proteins: roles in antioxidant defense and cellular regulation. IUBMB Life 2000 50(4-5):301-307

38. Moskovitz J, Singh VK, Requena J, Wilkinson BJ, Jayaswal RK, Stadtman ER: Purification and characterization of methionine sulfoxide reductases from mouse and Staphylococcus aureus and their substrate stereospecificity. Biochem Biophys Res Commun 2002, 290(1):62-65.

39. Rivera C, Gurard-Levin ZA, Almouzni G, Loyola A: Histone lysine methylation and chromatin replication. Biochim Biophys Acta 2014, (0): doi:10.1016/j. bbagrm.2014.03.009. [Epub ahead of print].

40. Van den Steen PE, Proost P, Grillet B, Brand DD, Kang AH, Van Damme J, Opdenakker G: Cleavage of denatured natural collagen type II by neutrophil gelatinase $B$ reveals enzyme specificity, post-translational modifications in the substrate, and the formation of remnant epitopes in rheumatoid arthritis. FASEB J 2002, 16(3):379-389.
41. Ivan M, Kondo K, Yang H, Kim W, Valiando J, Ohh M, Salic A, Asara JM, Lane WS, Kaelin WG Jr: HIFalpha targeted for VHL-mediated destruction by proline hydroxylation: implications for $\mathrm{O} 2$ sensing. Science 2001 292(5516):464-468

42. Lee I, Huttemann M: Energy crisis: the role of oxidative phosphorylation in acute inflammation and sepsis. Biochim Biophys Acta 2014, 1842(9):1579-1586.

43. Corpas FJ, Leterrier M, Begara-Morales JC, Valderrama R, Chaki M, LopezJaramillo J, Luque F, Palma JM, Padilla MN, Sánchez-Calvo B, Mata-Pérez C, Barroso JB: Inhibition of peroxisomal hydroxypyruvate reductase (HPR1) by tyrosine nitration. Biochim Biophys Acta 2013, 1830(11):4981-4989.

44. Bordoli L, Kiefer F, Arnold K, Benkert P, Battey J, Schwede T: Protein structure homology modeling using SWISS-MODEL workspace. Nat Protoc 2009, 4(1):1-13.

45. Zagotta WN, Olivier NB, Black KD, Young EC, Olson R, Gouaux E: Structural basis for modulation and agonist specificity of HCN pacemaker channels. Nature 2003, 425(6954):200-205.

46. Su Z, Limberis J, Martin RL, Xu R, Kolbe K, Heinemann SH, Hoshi T, Cox BF, Gintant GA: Functional consequences of methionine oxidation of hERG potassium channels. Biochem Pharmacol 2007, 74(5):702-711.

47. Monteiro HP, Arai RJ, Travassos LR: Protein tyrosine phosphorylation and protein tyrosine nitration in redox signaling. Antioxid Redox Signal 2008, 10(5):843-889

48. Dugave $C$, Demange L: Cis-trans isomerization of organic molecules and biomolecules: implications and applications. Chem Rev 2003, 103(7):2475-2532

49. Wang K, Wang Y, Yao M, Xu D: Diethyl 4-(2-meth-oxy-phen-yl)-2,6-dimethyl-1,4-di-hydro-pyridine-3,5-di-carboxyl-ate. Acta Crystallogr Sect E Struct Rep Online 2013, 69(Pt 5):0785.

50. Baker NA, Sept D, Joseph S, Holst MJ, McCammon JA: Electrostatics of nanosystems: application to microtubules and the ribosome. Proc Nat Acad Sci U S A 2001, 98(18):10037-10041.

\section{doi:10.1186/s12859-014-0370-6}

Cite this article as: Warnecke et al:: PyTMs: a useful PyMOL plugin for modeling common post-translational modifications. BMC Bioinformatics 2014 15:370

\section{Submit your next manuscript to BioMed Central and take full advantage of:}

- Convenient online submission

- Thorough peer review

- No space constraints or color figure charges

- Immediate publication on acceptance

- Inclusion in PubMed, CAS, Scopus and Google Scholar

- Research which is freely available for redistribution 